\title{
A novel image based end-member extraction technique to map green, non-photosynthetic and bare soil fractions using Landsat data
}

\author{
Peter Tan, Leo Lymburner and Norman Mueller \\ ${ }^{a}$ National Earth Observation Group (NEO), Geoscience Australia \\ Peter.Tan@ga.gov.au
}

\begin{abstract}
In most cases a single pixel in a satellite image contains information from more than one type of land cover substance. One challenge is to decompose a pixel with mixed spectral readings into a set of endmembers, and estimate the corresponding abundance fractions. The linear spectral unmixing model assumes that spectral reading of a single pixel is a linear combination of spectral readings from a set of endmembers. Most linear spectral unmixing algorithms rely on spectral signatures from endmembers in predefined libraries obtained from previous on-ground studies. Therefore, the applications of these algorithms are restricted to images whose extent and acquisition time coincide with those of the endmember library. We propose a linear spectral unmixing algorithm which is able to identify a set of endmembers from the actual image of the studied area. Existing spectral libraries are used as training sets to infer a model which determines the class labels of the derived image based endmembers. The advantage of such an approach is that it is capable of performing consistent spectral unmixing in areas with no established endmember libraries. Testing has been conducted on a Landsat7 ETM+ image subset of the Gwydir region acquired in Jun 2008. Three types of land cover classes: bare soil, green vegetation and non-photosynthetic are specified for this test. A number of ground abundance observations were obtained from a corresponding field trip. The study successfully identified a set of 256 endmember samples from the image for three specified land cover classes. For most test points, the spectral unmixing and estimation of the corresponding abundance are consistent with the ground validation data.
\end{abstract}

Keywords: Spectral unmixing, endmember, convex hull, linear programming 
Tan et al., A novel image based end-member extraction technique to map green, non-photosynthetic and bare soil fractions using Landsat data

\section{INTRODUCTION}

In literature of remotely sensed image analysis, an endmember is defined as a pixel containing only one land cover substance. However, due to limited resolution of the sensor, in most cases a single pixel in a satellite image contains more than one type of land cover substances. In many real-world applications based on extracting spatial information from remotely sensed images, one challenge is to decompose a pixel with mixed spectral readings into a set of endmembers and estimate the corresponding abundance fractions.

The underlying theory behind fractional cover mapping is that any land surface can be described as a mixture of different cover types (Keshava, 2003). For example a full dam is represented by a cover of $100 \%$ water. A field with an immature crop can be represented as having 50\% bare soil cover and $50 \%$ green vegetation cover. A grazing paddock could be represented by $15 \%$ green vegetation cover, $60 \%$ non-photosynthetic vegetation and $25 \%$ bare soil. Long term fractional cover dynamics provide critical insight into a wide range of phenomena, and they provide vital inputs into a range of biophysical models and decision support systems. However context is critical in interpreting fractional cover behaviour, because the same change in fractional cover can have dramatically different implications depending on the landscape and/or temporal context. For example a shift from $100 \%$ green fractional cover to $100 \%$ bare soil cover could represent a crop being harvested or a forest being cleared. Clearly, context is crucial in interpreting land cover behaviour and identifying an appropriate response (if any) that sudden or subtle changes in land cover. For the purposes of this study the ISO Land Cover classification is used to provide context.

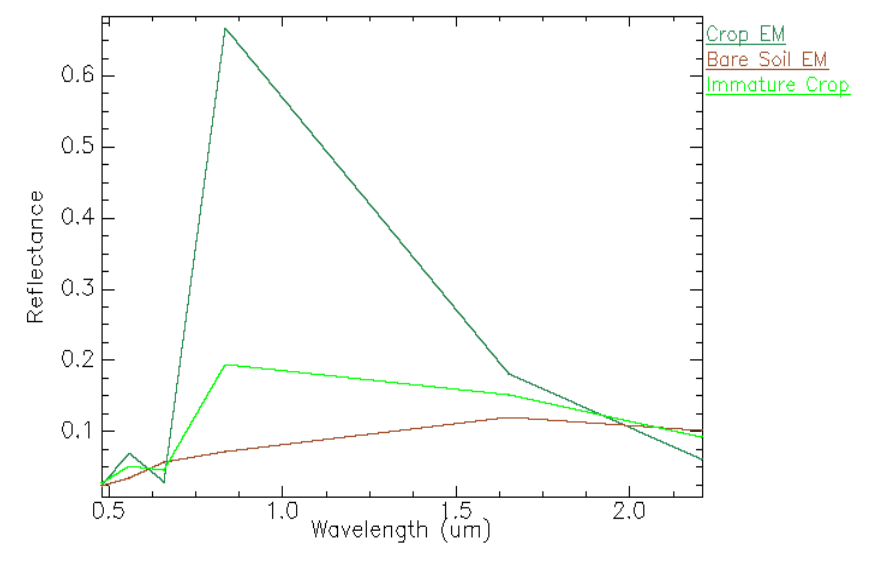

Figure $1100 \%$ green vegetation and $100 \%$ bare soil end members(EMs) and the reflectance of an immature $\operatorname{crop}(60 \%$ bare soil, $40 \%$ green vegetation)
The single biggest challenge in terms of estimating fractional cover information from remotely sensed imagery is the relationship between spectral reflectance and the cover fractions. In theory the spectral reflectance of a pure fractional cover i.e. $100 \%$ green vegetation or $100 \%$ bare soil will occupy a distinct part of spectral space. These reflectance curves that represent pure fractional cover types are often referred to as end members. Taking the example of an immature crop i.e. a mixture of green vegetation and bare soil then the fractional cover of both cover types can be estimated based on the similarity between the crop reflectance and one of those two end members (Figure 1). If the crop is near maturity, green cover fraction will approach one and the reflectance will look similar to the pure green cover fraction endmember. If the crop is immature then the bare soil fraction will be larger and the influence of the soil reflectance on the spectra for that pixel will be large. The spectra will therefore be more similar to the reflectance curve of the $100 \%$ bare soil end member.

The spectral unmixing models can be either linear or nonlinear (Keshava, 2003). Both types of models have their advantages and limitations. The linear spectral unmixing model assumes that the mixed spectral reading of a single pixel is a linear combination of spectral readings of a set of endmembers. As such, the linear spectral unmixing model also assumes independence between distinct bands and the interferences among bands are negative. On the other hand, the nonlinear models, such as regression trees algorithm (Borel \& Gerstl, 1994), do not make such assumptions. However, the complexities of nonlinear models are usually much higher compared to those of linear models. Therefore, nonlinear models are usually less robust, especially when a large set of training samples cannot be obtained.

Previous researches showed that linearity assumptions hold in most multi-spectral and hyper-spectral imagery analysis, especially for low and medium resolution remotely sensed images, where the scale of a single pixel is relatively large. In most scenarios, the spectral readings (often known as signatures) of endmembers, the number of endmembers and the corresponding abundance fractions are unknown. A typical linear spectral unmixing routine for a pixel usually consists of several steps described as follows. 
Tan et al., A novel image based end-member extraction technique to map green, non-photosynthetic and bare soil fractions using Landsat data

1. Identify a set of endmembers for a remotely sensed image and obtaining the signatures of the endmembers

2. Decide the number and the classes of endmembers within the pixel

3. Unmix spectral reading of the pixel with a subset of endmembers defined in the step 2

4. Estimate the corresponding abundance fraction for each endmember within the pixel

Many linear spectral unmixing algorithms (Hu, Lee, \& Scarpace, 1999) bypassed step one by importing a pre-defined endmember library. The signatures of endmembers in such a library are usually acquired from field surveys or previous studies in other regions. However, endmember signatures vary significantly in different conditions. Such variability can be summarised as follows:

- Spatial variability: endmember signatures do not have uniform readings spatially. For example, endmember signatures of the same type of green vegetation change gradually across different climate zones.

- Temporal variability: endmember signatures vary at different time of the year. For example, many types of green vegetation such as tree canopy or grass land exhibit significantly different characters in different seasons.

- Environmental variability: endmember signatures vary in different environmental contexts. For example, varying reflectance of bare soil under changing level of moisture is a well-known problem for remotely sensed scientists. Other environmental factors such as soil chemical and physical property also affect soil endmember signatures.

A large number of researches have been conducted on linear spectral unmixing algorithms with library-based endmembers. However, most of those researches were restricted in limited areas so that the region and the timing of studied imagery and those of the endmember library do not coincide. The purpose of this study is to devise generic linear spectral unmixing algorithms which can be applied to continental scale remotely sensed imagery analysis in an operational environment.

Image-based endmember extraction methods address endmember variability issues by identifying endmembers and obtaining their signatures from remotely sensed imagery. Such an approach is often preferred as field spectra in a pre-defined library are rarely acquired under the same conditions of remotely sensed imagery. Another advantage of using image-based endmembers is that the bias components in noise are cancelled out in the linear regressions as endmembers and pixels have independent and identically distributed (i.i.d.) bias.

\section{MATHEMATICAL MODELS AND ALGORITHMS}

In this analysis, a single pixel in a multi-spectral satellite image is regarded as a D-dimensional vector, where $\mathrm{D}$ is the number of spectral bands of the sensor. In such setting, a remotely sensed image is represented by a set of D-dimensional vectors. Every pixel in an image is unmixed as a linear combination of at most D+1 endmembers. There are constraints that the coefficients are non-negative and the sum of the coefficients equals to one.

In the proposed image-based endmember finding and linear unmixing method, a linear unmixing process is reframed as an optimization problem solved by standard linear programming procedures. The effectiveness and the speed of linear programming procedures depend on the quality and the size of the linear programming matrix. For this reason and to keep computational time in rein, an algorithm that dynamically updates the linear programming matrix is implemented. By only including the endmembers with large number of pixels being unmixed against, the size of the matrix is kept under a cap. In the initial stage, the program randomly selects a set of pixels as candidate endmembers. The program then consecutively unmixes pixels of the imagery and updates the endmember library by running the linear programming procedures.

The result of the analysis is represented as a set of (D-1)-dimensional simplex, each of which contains a subset of pixels from the image. The found endmembers are the vertices of the simplexes, which are the vectors assembling the edges of the simplexes. The abundances of land cover substances for a pixel are estimated by unmixing the pixel spectra against endmembers in the simplex where the pixel falls into. 
Tan et al., A novel image based end-member extraction technique to map green, non-photosynthetic and bare soil fractions using Landsat data

The proposed algorithm is implemented in a program written in $\mathrm{C}++$ and using CUDA GPGPU SDK.

\subsection{Convex hull problem}

Given a set $A \in R^{D}$ of points, if $C_{A}$ is the convex hull for $A$, then every point in $A$ can be expressed as a convex combination of at most $\mathrm{D}+1$ points in $C_{A}$. In geometry, the convex hull consists of extreme points of set $A$. A group of supporting hyperplanes constructed by connecting points in $\mathrm{C}_{\mathrm{A}}$ envelops all points in set $\mathrm{A}$. Figure 2 shows a convex hull problem in the 2-dimensional space. The black points are wrapped by the polygon created by connecting the red points in the convex hull.

For sets in low dimensional $(\mathrm{D}<=3)$ space, there are established algorithms to compute the convex hull for a given set of N points, such as Jarvis' algorithm, Graham's algorithm and Chan's algorithm (Chan, 1996). These algorithms are capable of solving the convex hull problem in low dimensional space in time

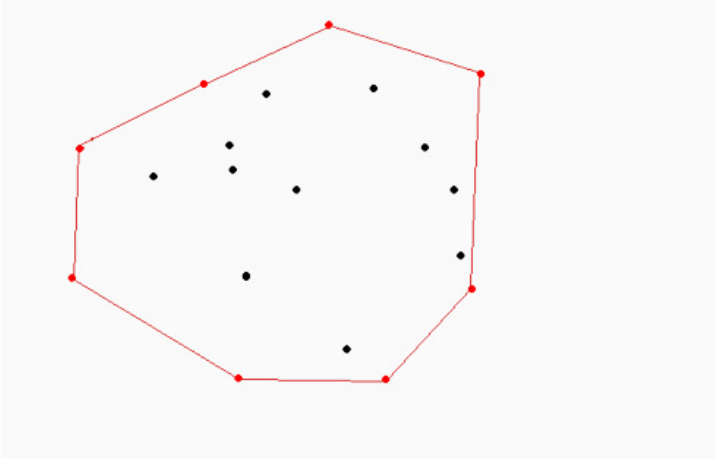

Figure 2 Convex hull problem $\mathrm{O}(N \log N)$. For sets in high dimensional space (D $>3$ ), the Quickhull algorithm (Barber, Dobkin, \& Huhdanpaa, 1996) returns the convex hull for a set in time $\mathrm{O}\left(N \log N+N^{\left[\frac{D}{2}\right]}\right)$. For the purpose of finding endmembers from a Landsat scene, with $\mathrm{N}$ approaching millions and $\mathrm{D}$ is equivalent to 6 , it is impractical to apply the Quickhull algorithm to identify the convex hull for a set of Landsat pixels. Therefore an algorithm to use linear programming techniques to approximate the convex hull for a set of Landsat pixels is proposed in section 2.3.

\subsection{Linear programming}

Linear programming (Dantzig, 1963) is an optimization process to achieve the optimal outcome of a predefined function under a given set of linear constraints. It is a specific case of mathematical optimization. A linear programming problem usually consists of a linear objective function and a set of linear constraints, which can include equalities and inequalities. Linear programming problems can be expressed in mathematical terms as follows.

Maximize $c^{T} x$

subject to $A^{T} x+s=b$

and $x \geq 0, s \geq 0$

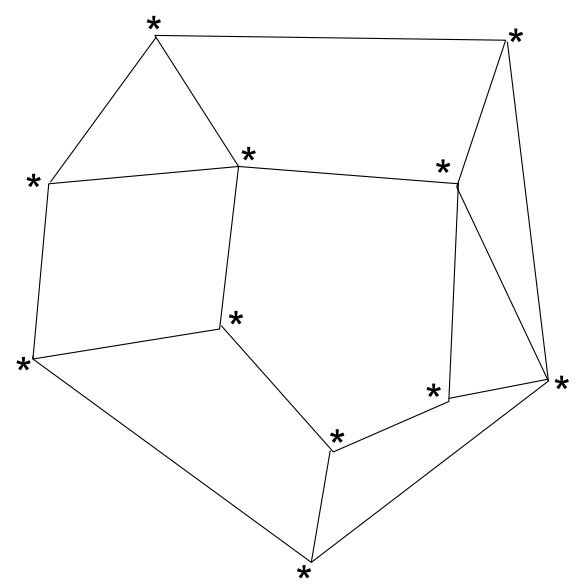

Figure 3 Vertices of a feasible polytope
Where $x$ and $s$ represent vectors to be determined in order to optimize the objective function, $b$ and $c$ are given vectors of coefficients, $A$ is a given matrix of coefficients. In geometry term, equalities and inequalities in (1) define a feasible region which is represented by a D-dimensional polytope. Following the fundamental theorem of linear programming, if solutions for (1) exist, then there is at least one solution is a basic optimal point, which is one of the vertices in the feasible polytope for the problem. Figure 3 shows such vertices of a feasible polytope.

The simplex method (Dantzig, 1963) (Nelder, 1965) was proposed by George Dantzig in 1947. It remains one of the most popular algorithms for solving linear programming problems. The simplex algorithm iterates among the vertices along the edges of the polytope (Press, Flannery, Teukolsky, $\&$ Vetterling, 1992). At each iteration, the direction of the move is guided by a sophisticated rule known as steepest 
Tan et al., A novel image based end-member extraction technique to map green, non-photosynthetic and bare soil fractions using Landsat data

edge, which chooses the direction resulting in the largest decrease $c^{T} x$ per unit among all candidates. The process continues until the optimal solution is found.

\subsection{Endmember identification and spectral unmixing}

Given a set of pixels, randomly divide the pixels into 2 sets: a set of candidate endmembers and the rest of the pixels are put into another set. Also define a set of counters $\left\{C_{j}, j \in[1, N]\right\}$, each of $C_{j}$ is corresponding to a vector in the candidate endmember set. The values $C_{j}$ in the counter set are set to zero initially.

Define a matrix $\mathrm{X}=\begin{array}{cccc}x_{1,1} & x_{2,1} & \cdots & x_{N, 1} \\ x_{1,2} & x_{2,2} & \ddots & x_{N, 2} \\ \vdots & \vdots & \ddots & \vdots \\ x_{1, D} & x_{2, D} & \cdots & x_{N, D}\end{array}$, every column is a vector corresponding to one candidate endmember pixel, where $\mathrm{D}$ is the number of spectral band and the $\mathrm{N}$ is the number of candidate endmember in the set. Define abundance vector $R=\left[\begin{array}{llll}r_{1} & r_{2} & \ldots & r_{N}\end{array}\right]^{T}$, the vector $Y=\left[\begin{array}{llllll}y_{1} & y_{2} & \ldots & y_{D}\end{array}\right]^{T}$ representing a target pixel to be unmixed. Then a linear programming problem to find endmembers for $\mathrm{Y}$ can be defined as follows.

Maximize $\sum_{i=1}^{N} r_{i}$, subject to $X R=Y, \sum_{i=1}^{N} r_{i} \leq 1, r_{i} \geq 0$.

1. Solve the linear programming problem using the simplex method, find the optimal abundance vector $R$ for pixel Y

2. Find the subset of the index numbers of the non-zero elements of $R$ and note the set as $\left\{\begin{array}{llll}I_{1} & I_{2} & \ldots & I_{D+1}\end{array}\right\}$

3. Update counter $C_{j}$ by $C_{j} \leftarrow C_{j}+1, j \in\left\{\begin{array}{llll}I_{1} & I_{2} & \ldots & I_{D+1}\end{array}\right\}$

4. Repeat step 1 to 3 for a set of $\mathrm{K}$ pixels, where $\mathrm{K}$ is a predefined number.

5. Order set $\left\{C_{j}, j \in[1, N]\right\}$ in descending order. Replace $\mathrm{M}$ vectors with the least value of $C_{j}$ in the matrix $\mathrm{X}$ with vectors either being unmixed unsuccessfully or with lower value of $\sum_{i=1}^{N} r_{i}$

6. Reset $C_{j}, j \in[1, N]$

7. Repeat step 1 to 6 until all pixels in the image have been unmixed

8. Reduce the size of the endmember set $\mathrm{X}$ using step 1 to 7

When the size of the endmember set $\mathrm{X}$ is reduced to a predefined number, then the endmember identification procedure ends. The vectors in the endmember set are then classified into 3 types of land cover endmembers: bare soil, green vegetation and non-photosynthesis vegetation. The classification model is created by training a nearest neighbour classifier using an existing endmember library. After the set of endmembers is classified, every pixel of the whole image was unmixed against the endmember set found in the procedure. The abundance of fractional cover for each class is then determined by the class labels of $\mathrm{D}+1$ endmember found by the spectral unmixing procedure.

\section{EXPERIMENTAL RESULTS AND DISCUSSIONS:}

Experiments had been conducted on part of a Landsat ETM+ image over Gwydir catchment area taken in Jun, 2008. The dimension of the test image is 2000 by 1500 pixels. So there are 3 million 6-dimension vectors in the data set. Three types of land cover endmembers: bare soil, green vegetation and nonphotosynthesis vegetation are specified for this test.

A field trip to the area which is covered by the image was also conducted at the same day. 24 sites were selected during the field trip. The abundances of fractional cover classes of the sites were visually estimated. The records were then compared to the experimental results from proposed spectral unmixing procedure. 
Tan et al., A novel image based end-member extraction technique to map green, non-photosynthetic and bare soil fractions using Landsat data

A set consisting of 256 endmember samples were extracted from the image. Then a classifier using nearest neighbours algorithm were inferred from an existing endmember library. For most of the image, the spectral unmixing and the estimation of the corresponding abundance are consistent with the field observation data. Table 1 shows a comparison between the fractional ground cover estimations made by the proposed scheme and the field observations for the 24 test sites. Figure 4 and 5 are X-Y plots of the ground truth and the estimations of the bare soil and non-photosynthesis fractions (in percentage).

Table 1 Fractional ground cover estimations compared to estimations by field observation (bare soil is noted as BS, while green vegetation and non-photosynthesis vegetation are noted as GV and NPV respectively)

\begin{tabular}{|c|c|c|c|c|c|}
\hline \multicolumn{2}{|c|}{ Field Inspection (\%) } & \multicolumn{4}{|c|}{ Spectral Unmixing Algorithm (\%) } \\
\hline BS & $\mathrm{GV}$ & NPV & BS & GV & NPV \\
\hline 20 & 0 & 80 & 33 & 3 & 64 \\
\hline 30 & 0 & 70 & 47 & 1 & 52 \\
\hline 40 & 0 & 60 & 44 & 3 & 53 \\
\hline 80 & 0 & 20 & 63 & 5 & 31 \\
\hline 80 & 0 & 20 & 44 & 4 & 52 \\
\hline 15 & 85 & 0 & 6 & 88 & 6 \\
\hline 90 & 0 & 10 & 74 & 1 & 25 \\
\hline 90 & 0 & 10 & 74 & 1 & 25 \\
\hline 10 & 10 & 80 & 25 & 26 & 48 \\
\hline 20 & 60 & 20 & 5 & 92 & 4 \\
\hline 50 & 25 & 25 & 36 & 9 & 55 \\
\hline 30 & 0 & 70 & 40 & 0 & 60 \\
\hline 50 & 0 & 50 & 45 & 4 & 52 \\
\hline 5 & 95 & 0 & 6 & 92 & 2 \\
\hline 5 & 95 & 0 & 2 & 98 & 0 \\
\hline 70 & 15 & 15 & 52 & 7 & 42 \\
\hline 40 & 0 & 60 & 40 & 1 & 59 \\
\hline 30 & 0 & 70 & 52 & 9 & 40 \\
\hline 70 & 0 & 30 & 37 & 17 & 45 \\
\hline 30 & 0 & 70 & 49 & 9 & 42 \\
\hline 33 & 33 & 33 & 18 & 10 & 71 \\
\hline 50 & 25 & 25 & 48 & 9 & 44 \\
\hline 20 & 0 & 80 & 36 & 2 & 50 \\
\hline 20 & 0 & 80 & 44 & 3 & 53 \\
\hline
\end{tabular}

There are inconsistencies of estimations of land cover abundance occurring in some parts of the image. Those inconsistent estimations are caused by incorrectly classified endmembers. When an endmember falls into some regions in the 6-dimensional space defined the problem, especially those near the decision boundaries, the probability of the inferred classifiers incorrectly classify it is high. In other words, some ground objects may not be easily put into one of the three pre-defined categories.

\section{CONCLUSION}

An image based endmember extraction scheme using linear programming technique to approximate the convex hull of a set of multi-spectral data was proposed. The proposed endmember identification scheme for Landsat 5/7 image data has successfully identified endmember pixels within the image. Experimental results show the spectral unmixing results in the image are consistent with estimations made by field observations.

One of future research directions is to cluster pixels with similar endmembers: pixels unmixed against by similar set of endmembers have homogenous land cover classes. Results of preliminary experiments had shown that such clusters discriminate areas with distinct ecological characteristics. Another possible way to forward is to integrate spatial-spectral information: objects can be created by grouping spatially correlated pixels into clusters and linear spectral unmixing is performed on the average spectra of the clusters. 
Tan et al., A novel image based end-member extraction technique to map green, non-photosynthetic and bare soil fractions using Landsat data

NPV

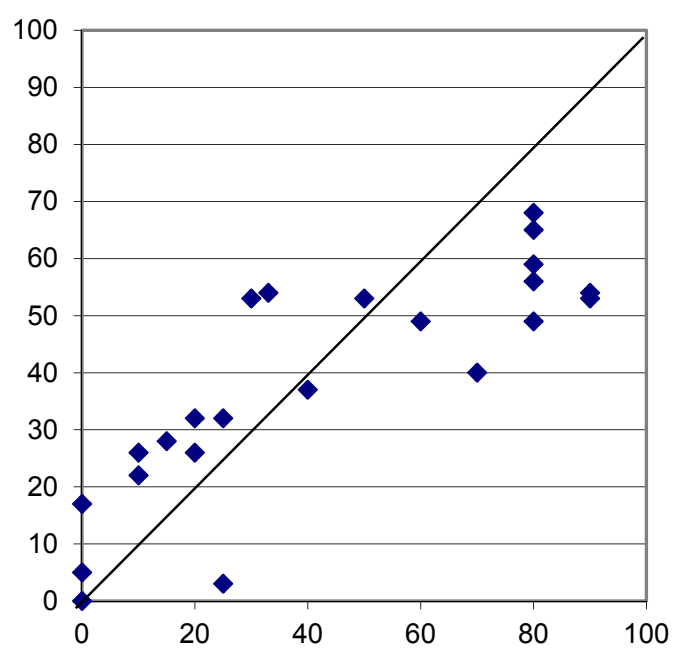

Figure $4 \mathrm{X}-\mathrm{Y}$ plot of ground truth and estimation of non-photosynthesis fractions
BS

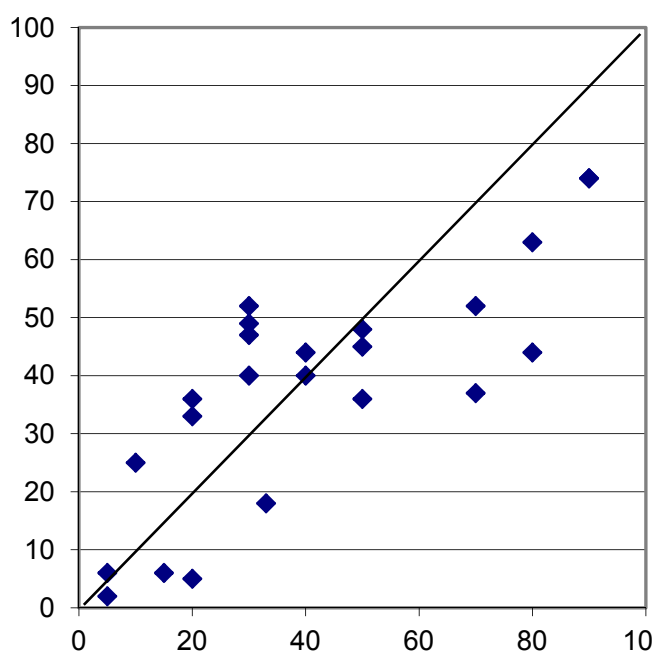

Figure $5 \mathrm{X}-\mathrm{Y}$ plot of ground truth and estimation of bare soil fractions

\section{ACKNOWLEDGMENTS}

This paper is published with the permission of the CEO, Geoscience Australia.

\section{REFERENCES}

Barber, C. B., Dobkin, D. P., \& Huhdanpaa, H. T. (1996). The Quickhull Algorithm for Convex Hulls. ACM Trans. Mathematical Software(22), 469-483.

Borel, C., \& Gerstl, S. (1994). Nonlinear spectral mixing models for vegetative and soil surfaces,. Remote Sensing of Environment, 47(3), 403-416.

Chan, T. (1996). Optimal Output-sensitive Convex Hull Algorithms in Two and Three Dimensions. Discrete \& Computational Geometry(16), 361-368.

Dantzig, G. B. (1963). Linear Programming and Extensions. Princeton, NJ: Princeton University Press.

Hu, Y.-H., Lee, H. B., \& Scarpace, F. L. (1999, Jan). Optimal linear spectral unmixing," , vol.37, no.1, pp.639,644, Jan 1999. IEEE Transactions on Geoscience and Remote Sensing, 37, 639-644.

Keshava, N. (2003). A Survey of Spectral Unmixing Algorithms A Survey of Spectral Unmixing. Lincoln Laboratory Journal, 14(1), 55-78.

Nelder, J. A. (1965). A Simplex Method for Function Minimization. Computer Journal(7), 308-313.

Press, W. H., Flannery, B. P., Teukolsky, S. A., \& Vetterling, W. T. (1992). Downhill Simplex Method in Multidimensions, in Numerical Recipes in FORTRAN: The Art of Scientific Computing (2nd ed.). Cambridge, England: Cambridge University Press. 